\title{
Recent results in Color Superconductivity
}

\section{G. Nardulli}




\section{QCD Phase at $\mathrm{T}=0$, large $\mu$}

Gluonic interaction between two quarks

$$
3 \times 3=\overline{3}+6
$$

The color antisymmetric $\overline{3}$ channel is attractive. Therefore Cooper's theorem implies formation of a condensate of Cooper pairs of quarks (diquarks) and color superconductivity

$$
\langle\psi \psi\rangle=\Delta \neq 0
$$




\section{Color Superconductivity at extreme densities}

- $\quad \mathbf{S}=\mathbf{0}$ state is preferred as more phase space available

- Pauli principle implies antisymmetry in flavor

- Asymptotically (very large densities):Color Flavor Locking (CFL; Alford, Rajagopal, Wilczek)

$$
\left.<0 \mathrm{I} \Psi_{\alpha}^{\mathrm{i}} \Psi_{\beta}^{\mathrm{j}} 10\right\rangle=\Delta \varepsilon^{\mathrm{ij} \gamma} \varepsilon_{\alpha \beta \gamma}
$$

Valid for $\mu \gg m_{q}$

Rigourously proved in QCD, albeit at very large $\mu$.

At intermediate densities, relevant for astrophysical applications the ground state is still unknown

Model calculations (NJL) as QCD unreliable 


\section{Deviations from CFL due to $s$ quark mass} effect.

Color and electric neutrality must be imposed;

$=>$ different gaps for quarks of different flavor.

Gapless phases uniform (gCFL) or not uniform (LOFF).

\section{Choosing the true vacuum}

The true ground state has the minimum value of the free energy (grand potential)

$$
\Omega=-\frac{1}{2 \pi^{2}} \int_{0}^{\Lambda} d p p^{2} \sum_{j=1}^{9}\left|\epsilon_{j}\left(\ell_{\|}\right)\right|+\frac{1}{G}\left(\Delta_{1}^{2}+\Delta_{2}^{2}+\Delta_{3}^{2}\right)-\frac{\mu_{Q}^{4}}{12 \pi^{2}}
$$




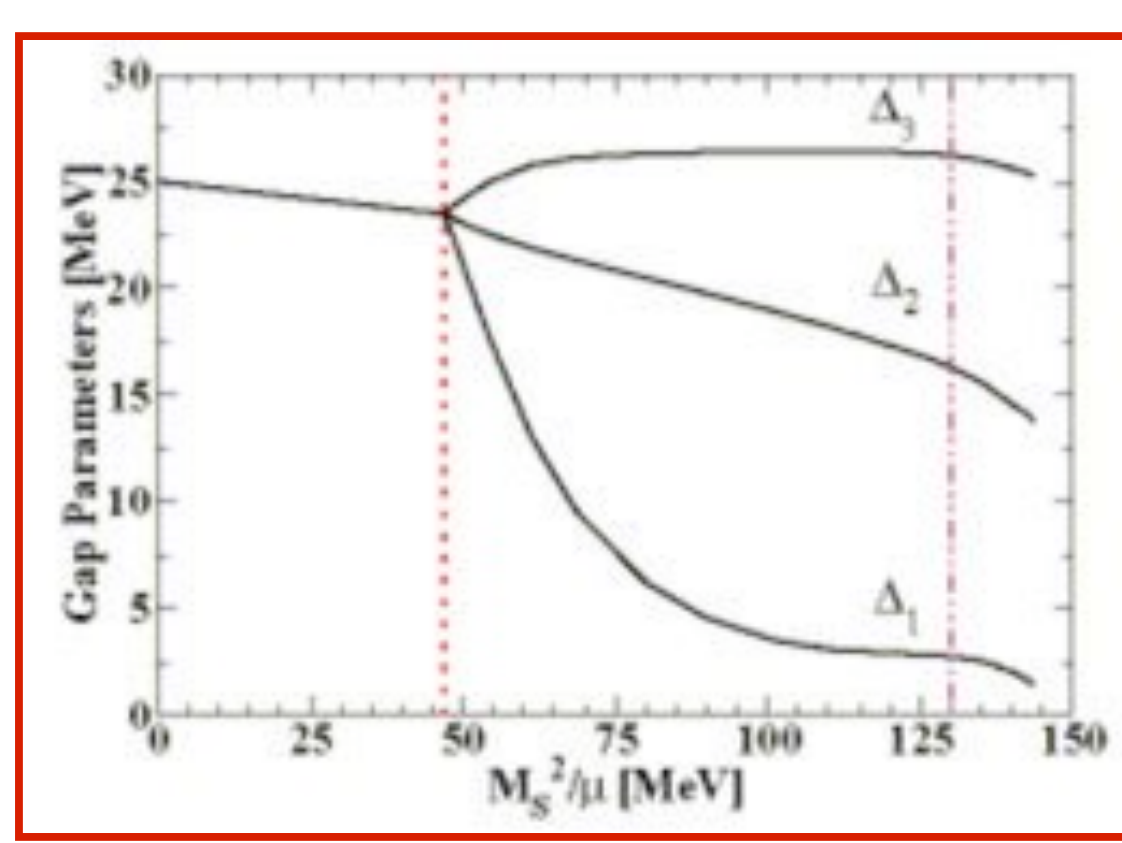

\section{Results for gCFL phase}

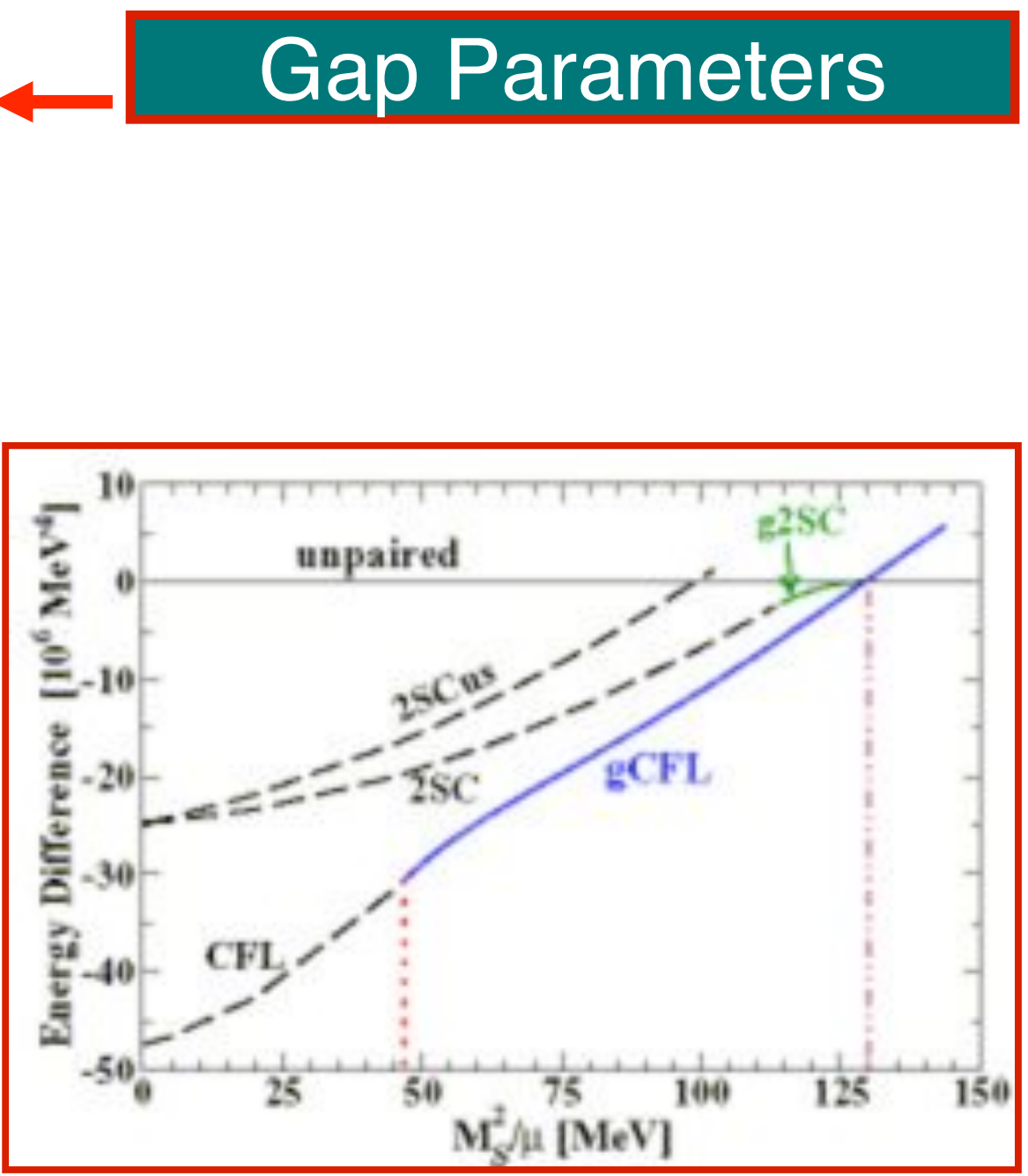

M.Alford, P.Jotwani, C.

Kouvaris, J. Kundu, K.Rajagopal 


\section{The importance of being gapless}

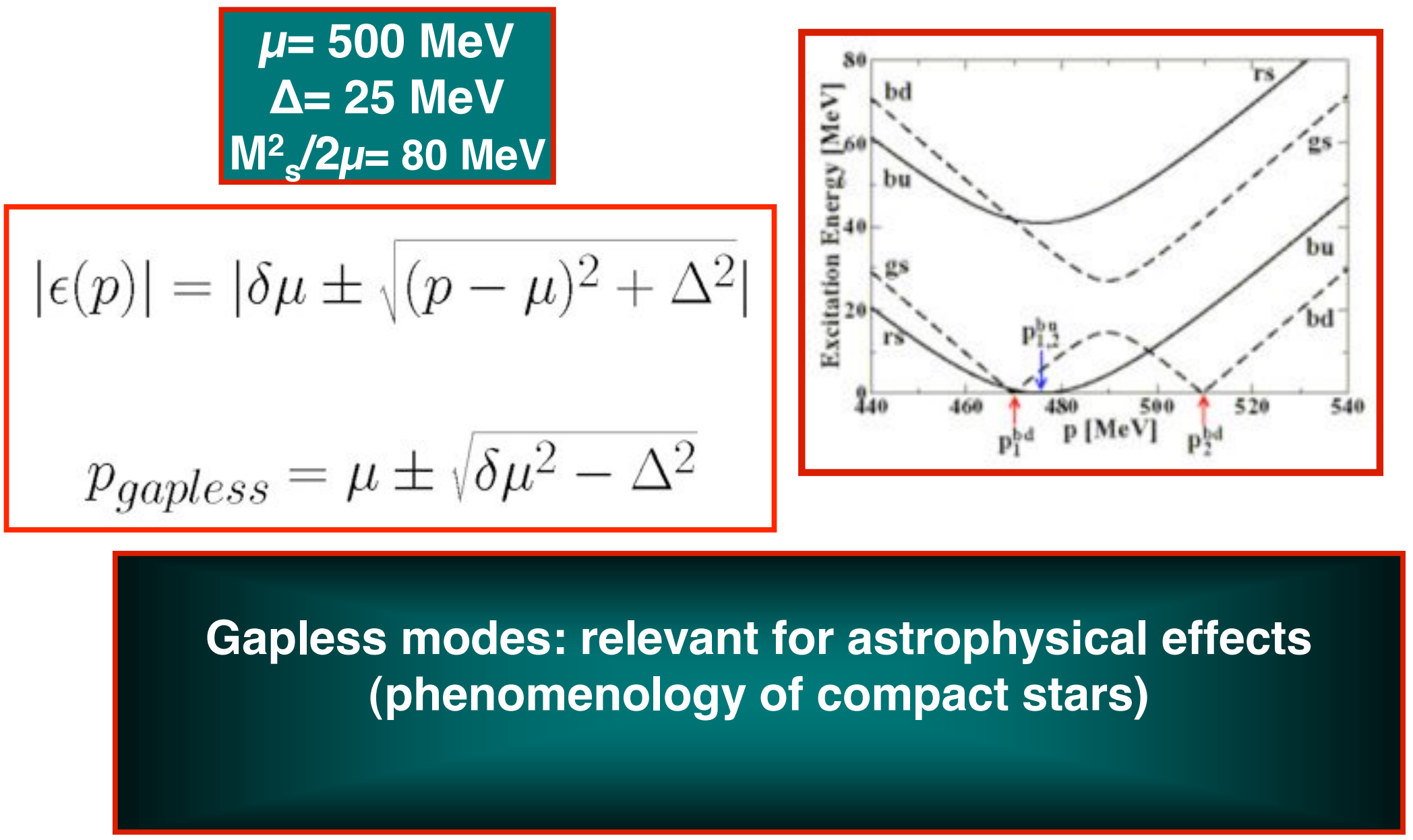




\section{Meissner masses in the gCFL phase}

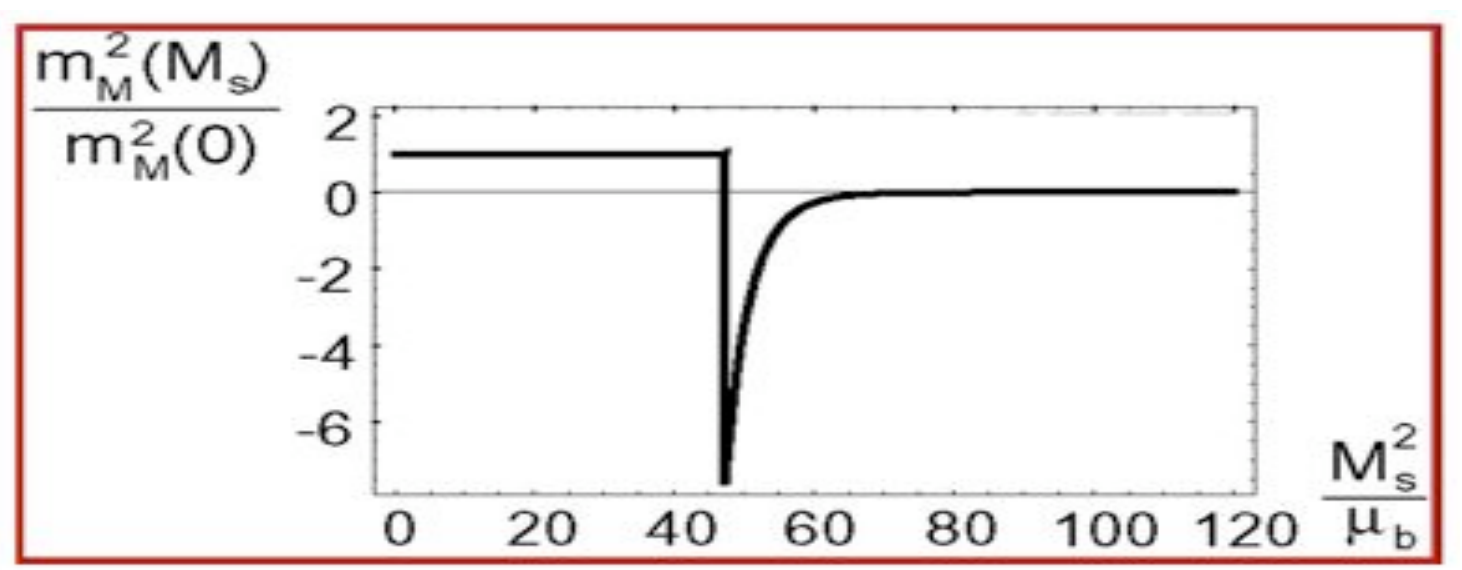

Solid line $a=1,2$; One Gluon"3mixed8": same behavior; others: no instability One $U_{e m}(1)$ : massless R. Casalbuoni, M. Mannarelli, G. N., M. Ruggieri, R. Gatto

Imaginary masses: instability of chromomagnetic type $\mathrm{gCFL}$ is not the true vacuum

Similar results for 5 gluons of broken generators in 2-flavor (Huang-Shovkovy)

LOFF phase favored (Giannakis-Ren, Fukushima) 


\section{Loff Phase: two flavors $(u, d)$}

If $\delta \mu \neq 0$ it can be energetically favourable to have states where the Cooper pair has total momentum $2 q \neq 0$

\section{LOFF= Larkin-Ovchinnikov\&} Fulde-Ferrell (1964); Alford, Bowers, Rajagopal (2000) R. Casalbuoni, G.N. Rev. Mod. Phys.(2004)

Studied in condensed matter (2D superconductors), cuprates

$$
\Delta(r)=<0 \text { I } \Psi(r) \Psi(r) \mid 0>=\Delta \exp \left[i\left(p_{1}+p_{2}\right) r\right]
$$




\section{LOFF phase: three flavors}

Needed for realistic calculations

- R. Casalbuoni, R.Gatto,,N.Ippolito,GN, M.Ruggieri : Ginzburg Landau expansion of the gap equation and free energy

- (Almost) complete analysis beyond GL: M.Mannarelli, K. Rajagopal, R. Sharma 


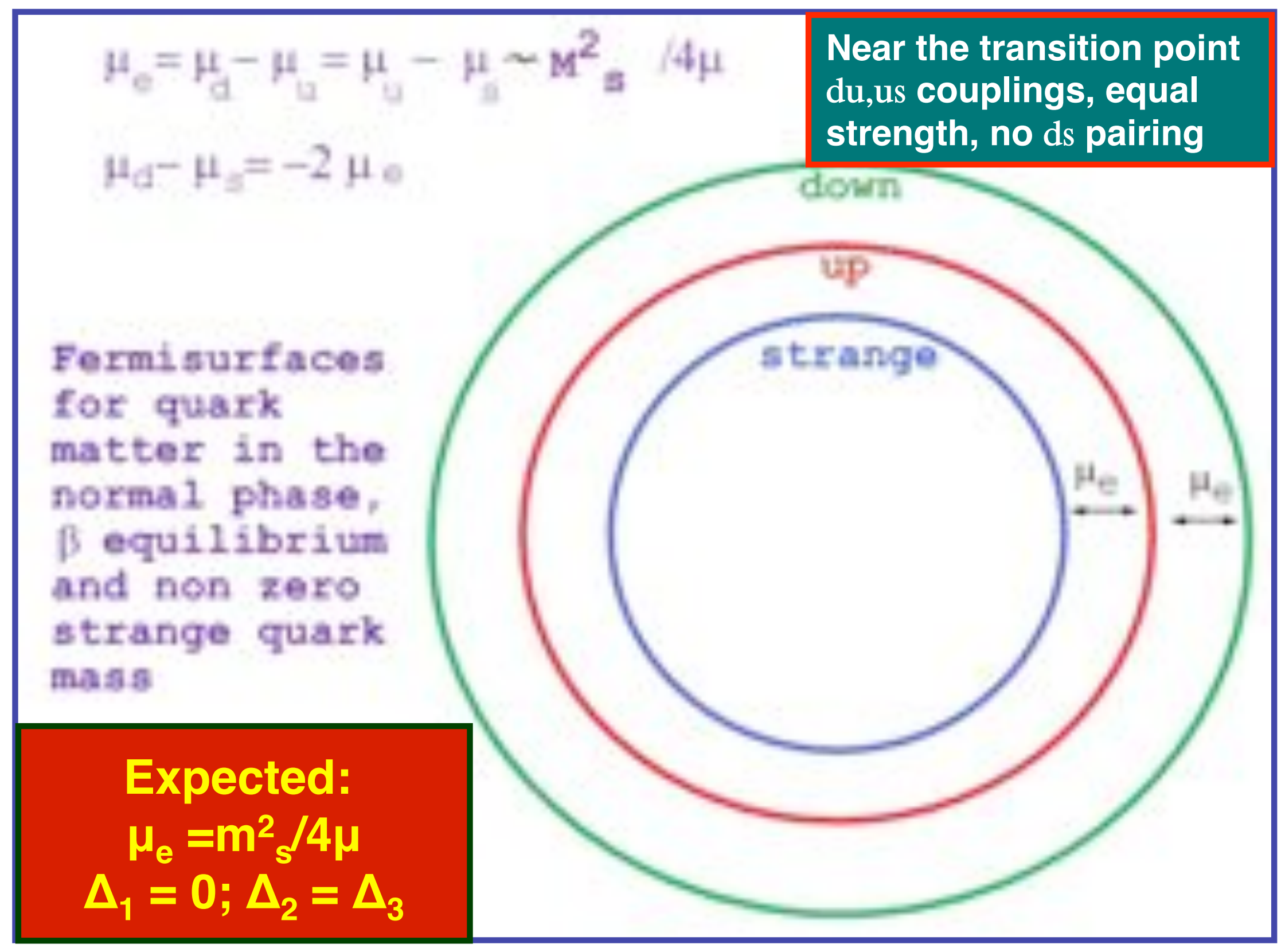




\section{Gap parameters}

$$
\Delta_{K}(r)=\Delta_{K} e^{2 i q_{k} r}
$$

For each inhomogeneous pairing a Fulde-Ferrell ansatz;

$$
2 q_{k}
$$

represents the momentum of the Cooper pair.

This is the simplest ansatz, other structures should be examined

Three independent functions
$\Delta_{1}(r)$,
$\Delta_{2}(r)$,
$\Delta_{3}(\mathbf{r})$

describing respectively $d-s$, u-s and u-d pairing. 


\section{$q_{2} q_{3}$ parallel favored by phase space}

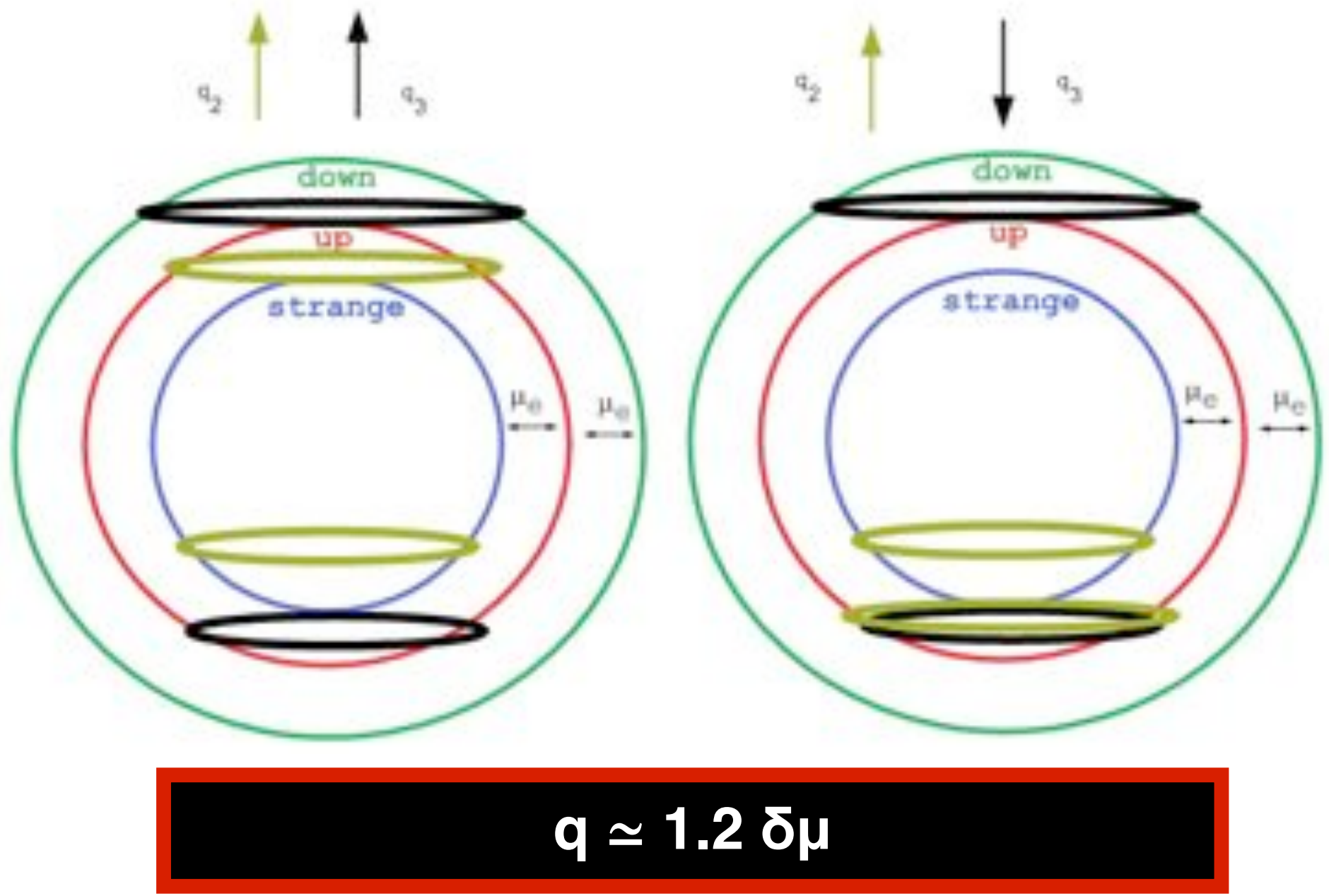

Nardulli, Minneapolis 05/12/06 


\section{Ginzburg-Landau expansion}

$$
\begin{gathered}
\Omega=\Omega_{\mathrm{n}}+1 / 4 \sum_{\mathrm{l}=1}{ }^{3}\left(2 \alpha_{1} \Delta_{1}^{2}+\beta_{1} \Delta_{1}^{4}+\right. \\
\left.\sum_{\mathrm{J} \neq \neq} \beta_{\mathrm{I}} \Delta_{1}^{2} \Delta_{\mathrm{J}}^{2}\right)+O\left(\Delta^{6}\right)
\end{gathered}
$$

$$
\Omega_{n}=-3 / 12 \pi^{2}\left(\mu_{u}{ }^{4}+\mu_{d}{ }^{4}+\mu_{s}{ }^{4}\right)-1 / 12 \pi^{2} \mu_{e}{ }^{4}
$$

$-\partial_{\Delta_{k}} \Omega=0$ minimum in $\Delta_{\kappa}$

R. Casalbuoni, R.Gatto,

N.Ippolito,GN,

M.Ruggieri

$-\partial_{\mu_{\mathrm{e}}} \Omega=0$ electrical neutrality

Approximation: $\mu_{3} \simeq \mu_{8} \simeq 0$ (confirmed by R.Ciminale et al., in preparation: Valid near the transition point, since this is the result for the normal phase)

The numerical results in this regime confirm $\Delta_{1}=0 ; \Delta_{2} \simeq \Delta_{3}$ 


\section{(Almost) complete calculation}

Assume from the very beginning

$$
\begin{aligned}
& \Delta_{1}=0 ; \Delta_{2}=\Delta_{3} \\
& \mu_{3}=\mu_{8}=0 ; \quad \mu_{e}=m_{s}^{2} / 4 \mu
\end{aligned}
$$

Since

$$
k_{u}+k_{d}=2 q_{3} ; k_{u}+k_{s}=2 q_{2}
$$

Shift momenta so that

$$
P_{u}=p=\mu v ; P_{d}=p+2 q_{3} ; P_{s}=p+2 q_{2}
$$

Corresponding to redefinitions of $\delta \mu$ :

$$
\delta \mu \rightarrow \delta \mu \pm q v
$$




\section{Comparison among different CSC phases}

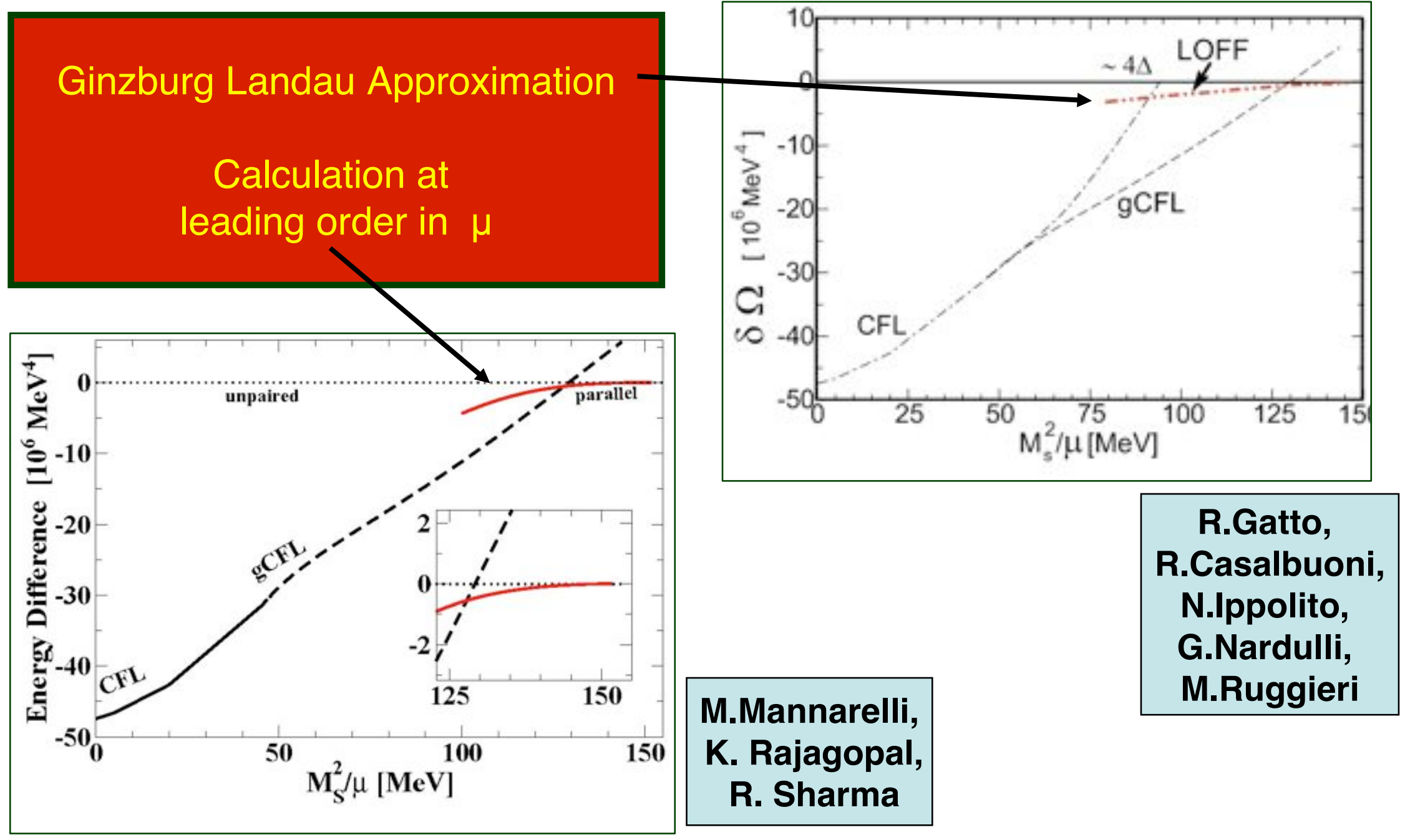

Nardulli, Minneapolis 05/12/06 


\section{Comparison between the two approaches}
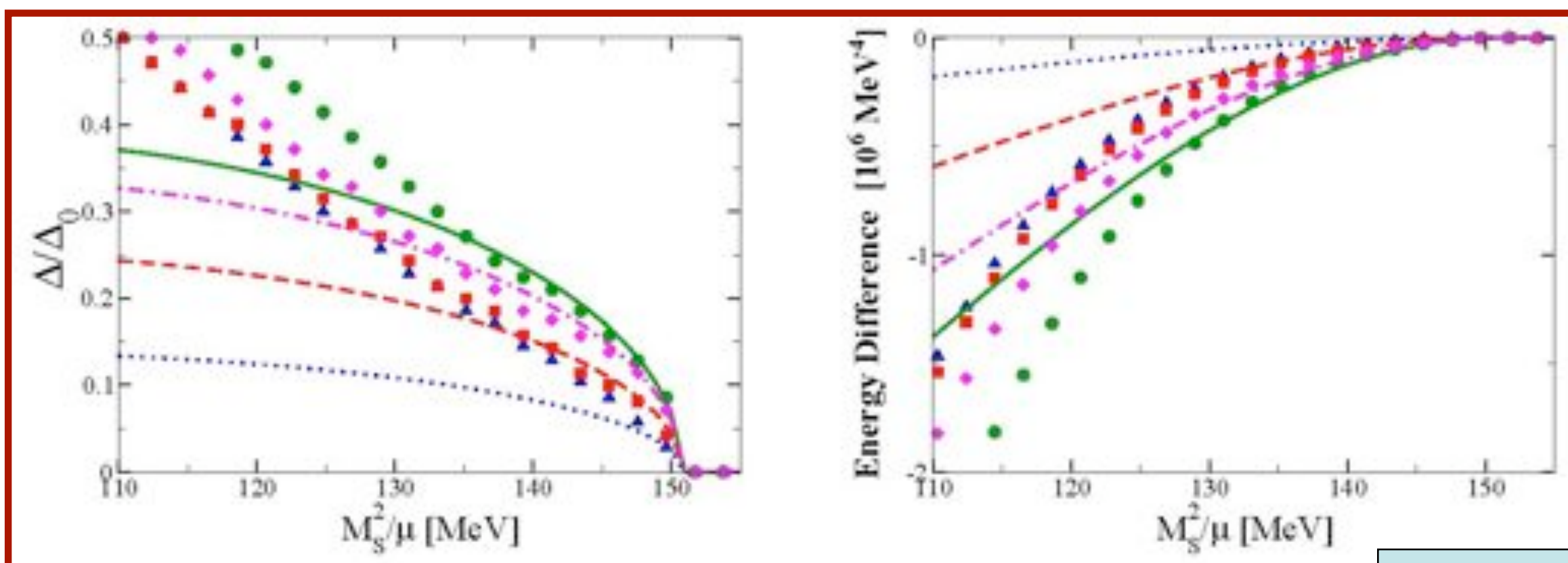

M.Mannarelli, K. Rajagopal,

Lines: GL. Dots: complete R. Sharma Green $=q_{2} q_{3}$ parallel. Magenta: $2 / 3 \pi$. Red; 7/8rr. Blue: $31 / 32 \pi$ 


\section{Chromomagnetic instability}

In gCFL : imaginary gluon Meissner masses

Studied in LOFF model with 2 flavors (Giannakis\&Ren, Fukushima, Gorbar, Hashimoto\&Miransky).

LOFF favored in comparison to gapless uniform conductive states. At least in the GL region (small gap) no chromomagnetic instability

For 3 flavors: recent results (Ciminale,Gatto,GN,Ruggieri) 


\section{Feynman Diagrams at $\mathrm{O}\left(\Delta^{4}\right)$}

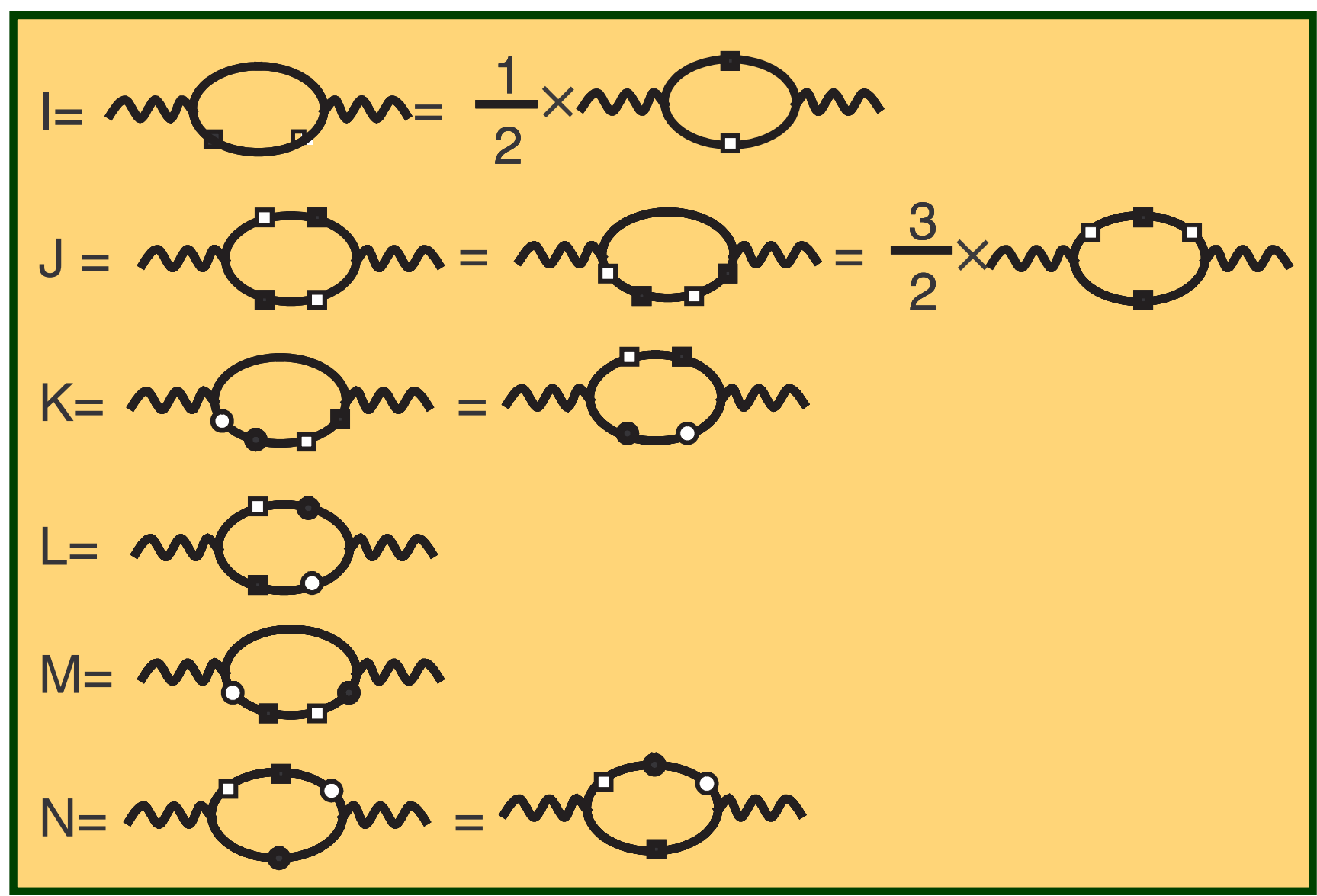

Nardulli, Minneapolis 05/12/06 


\section{Gluon Meissner masses:no instability}

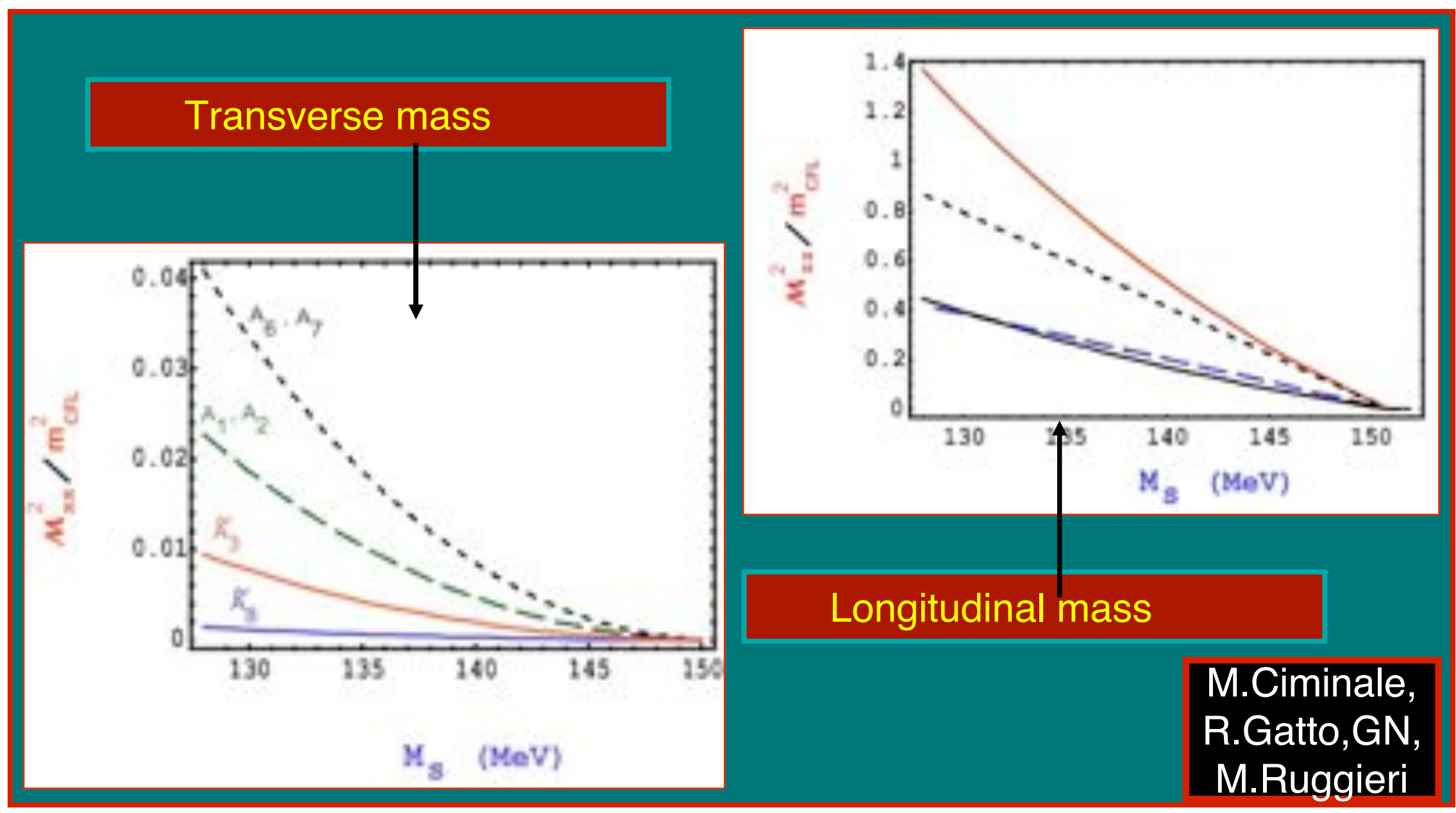




\section{Conclusions}

- QCD at intermediate densities, small T : active field of research

- Structure of vacuum not yet understood, though color superconductivity expected

- Likely gapless superconductivity, e.g. LOFF anisotropic state

- Results interesting for the effects on cooling of compact stars with a quark core: faster cooling 